\title{
Review Article \\ Electronic Nose Technologies in Monitoring Black Tea Manufacturing Process
}

\author{
Tharaga Sharmilan $\mathbb{D},{ }^{1,2}$ Iresha Premarathne, ${ }^{2}$ Indika Wanniarachchi $\mathbb{D},{ }^{3}$ Sandya Kumari, \\ and Dakshika Wanniarachchi $\mathbb{D D}^{2}$
}

\author{
${ }^{1}$ Department of Engineering Technology, Faculty of Technology, University of Sri Jayewardenepura, Sri Lanka \\ ${ }^{2}$ Instrument Center, Faculty of Applied Sciences, University of Sri Jayewardenepura, Sri Lanka \\ ${ }^{3}$ Department of Physics, Faculty of Applied Sciences, University of Sri Jayewardenepura, Sri Lanka \\ ${ }^{4}$ Department of Science \& Technology, Uva Wellassa University, Sri Lanka
}

Correspondence should be addressed to Dakshika Wanniarachchi; dakshikacw@sjp.ac.lk

Received 22 November 2019; Revised 18 March 2020; Accepted 17 June 2020; Published 9 July 2020

Academic Editor: Yuan Li

Copyright (c) 2020 Tharaga Sharmilan et al. This is an open access article distributed under the Creative Commons Attribution License, which permits unrestricted use, distribution, and reproduction in any medium, provided the original work is properly cited.

\begin{abstract}
"Tea" is a beverage which has a unique taste and aroma. The conventional method of tea manufacturing involves several stages. These are plucking, withering, rolling, fermentation, and finally firing. The quality parameters of tea (color, taste, and aroma) are developed during the fermentation stage where polyphenolic compounds are oxidized when exposed to air. Thus, controlling the fermentation stage will result in more consistent production of quality tea. The level of fermentation is often detected by humans as "first" and "second" noses as two distinct smell peaks appear during fermentation. The detection of the "second" aroma peak at the optimum fermentation is less consistent when decided by humans. Thus, an electronic nose is introduced to find the optimum level of fermentation detecting the variation in the aroma level. In this review, it is found that the systems developed are capable of detecting variation of the aroma level using an array of metal oxide semiconductor (MOS) gas sensors using different statistical and neural network techniques (SVD, 2-NM, MDM, PCA, SVM, RBF, SOM, PNN, and Recurrent Elman) successfully.
\end{abstract}

\section{Introduction}

Black tea is the most commonly consumed beverage in the word. The world production of black tea is about 3000 tons per year [1]. Quality parameters of tea such as liquor color, state, and mouth feel are evaluated by "tea tasters" when deciding market price for tea. A higher price for tea can only be guaranteed if the manufacturing process was conducted with the highest quality. This includes quality tea leaves plucked from plantations and proper factory conditions.

Stages in the black tea manufacturing process are plucking, withering, rolling, fermentation, firing, and packaging. The plucked tea leaves are dried to remove moisture content during the withering stage [2] and fed into the rollers. Tea particles after the rolling step are loaded to a rotorvane to separate smaller tea particles from larger pieces. The first set of smaller tea particles is called the "first dhool" and directly sent for fermentation. Then, larger particles undergo the rolling step again and are loaded back to the rotarvane to separate. The second set of smaller particles is called the "second dhool," and the tea particles from a third repeat of this cycle are called the "third dhool" [3]. Then, these classified leaves are laid on a tea bed to expose to oxygen during the fermentation process. In the presence of oxygen, chemical constituents in tea leaves react and produce polyphenol compounds and aromatic compounds [4]. Therefore, quality characteristics such as color, aroma, and taste of the tea are produced in this stage. Theaflavin (TF) and thearubigin (TR) are the main oxidized chemical compounds responsible for the brightness and briskness of the tea infusion while 
increasing mouth feel, color, and body of the tea infusion [5]. After that, fermented tea leaves are sent to the dryers. Then, dried leaves are sorted and packaged.

The fermentation stage is the most important among other stages [6-8] because final tea quality characteristics are developed at this stage. Fermentation time directly relates to the chemical changes during the fermentation process. Therefore, fermentation time has a significant impact on the final tea quality [9]. Since over and low fermentation leads to lack of final tea quality, optimum fermentation time should be identified to maintain high quality of black tea.

The optimum fermentation time is found in almost all tea factories based on the decision of the human detecting aroma and color changes of tea leaves. These decisions are very subjective. In general, it is accepted that the best quality tea will have a TF/TR ratio $1: 10$ [10] measured using spectrometric methods, and it was used for many decades as the main quality controlling parameter $[11,12]$. However, this method is not practical to analyze the fermentation level in real time due to time-consuming chemical analysis.

Therefore, development of a system which can detect the optimum fermentation level without use of chemicals and minimum human intervention is needed. Innovative technologies were developed by several research groups using an electronic eye method which analyzes images of tea leaves during fermentation to notice color changes [3, 12-17]. The second method involves an electronic nose system which detects aroma levels of tea particles during fermentation [18-32]. Recent studies focused on the combination of electronic eye and electronic nose methods [32-35] which are aimed at minimizing human intervention of observing color changes and smelling of tea particles. However, adopting these developed methods into the real factory condition is challenging as most of the studies are conducted in a laboratory scale [36]. There are very few studies conducted under factory conditions $[26,27]$.

Recently, the use of the e-nose technique for tea quality evaluation has increased. Therefore, a comparison of current systems and identification of drawbacks are important in developing novel technologies. Therefore, this paper will focus on the comparison of methods developed for analysis of tea aroma during the fermentation stage using "e-nose" systems under laboratory conditions and factory scale. In this paper, a brief discussion on chemical compounds detected in tea aroma followed by conventional methods used for detection is also discussed. Then, existing e-nose technologies and statistical algorithms which were used in data analysis will be discussed.

\section{Tea Quality Evaluation}

2.1. Aroma Compounds Released during Fermentation Process. When developing an e-nose system, it is important to identify different chemical compounds present in tea aroma. Enzymes catalyze oxidation of fatty acid, phenylalanine, terpenoids, and carotenoid compounds in tea leaves when exposed to air [37-39]. Oxidation of fatty acids results in grassy smelling compounds such as hexanol and hexanal (alcohols and aldehydes containing 6-9 carbon atoms) $[37,38]$. The extent of volatile compounds present depends on the climatic conditions as activity of lipoxygenase (LOX) and alcohol dehydrogenase enzymes is influenced by the temperature of the processing time. In fully oxidized tea and semioxidized tea, geraniol, linalool, and linalool oxides are major contributors to the overall aroma profile $[37,38]$. These floral and sweet smelling compounds are derived by geranyl pyrophosphate (GPP) which is degraded by geraniol synthase and linalool oxidase enzymes. Phenylethanol (flowery), phenylacetaldehyde (honey), and benzyl alcohol (sweet, fruity) are another group of compounds derived from phenylalanine. These compounds also contribute to the floral smell in aroma profile. Oxidation of $\beta$-carotenoids results in woody smell compound $\beta$-ionone and damscone. These contribute to the aroma compounds with C9-C13 aroma. The variation of carotenoid content in tea leaves subject to climatic, clone variety, and processing conditions results in variations in aroma profile. It was reported that high carotene content yields enhanced volatile flavor compounds [40, 41]. There are some biochemical compounds mainly responsible for the tea aroma and flavor, such as linalool, geraniol, phenyl acetaldehyde, benzaldehyde, methyl salicylate, and hexanal [37, 38, 40, 42] which are mostly aldehydes, ketones, esters, hydrocarbons, and furans [15].

In most of the factories, fermentation is continued until appearance of two distinct smell peaks with time. These are commonly denoted as "first nose" and "second nose" which can be identified only by highly trained factory personnel [10]. The first nose corresponds to a rawer, grassy smell of tea leaves while a fruity smell of tea leaves is prominent when tea particles turned to a more copper color. Table 1 indicates that prominent compounds correspond to each smell peak and their biochemical origin.

Therefore, when developing the "e-nose" system, it is important to choose sensors which are responsive to aldehydes and ketones with excellent sensitivities to detect subtle changes such as "first nose" and "second nose" as practiced in the factory.

2.2. Conventional Methods Used to Analyze Tea Aroma. Identification of aroma compounds present in tea is done with gas chromatography mass spectrometry (GCMS) and their characteristics (floral, grassy, sweet, etc.) using Gas Chromatography-Olfactometry (GC-O) [37, 38, 40-42]. GCMS has high sensitivity, and over 70 different chemical compounds present in tea aroma were identified in different studies [10, 37, 38, 40-56]. However, GCMS instruments are not found in almost all tea factories due to high price of the instrument. Since oxidation will continue on the way from the factory to the lab, GCMS has limitations to adapt to regular quality checking in the production line. Nowadays, these conventional techniques are used to compare the results of electronic devices when calibration is required.

2.3. Electronic Nose Technologies. Humans can detect different odors by special receptors located in the nasal cavity. These are olfactory cells located in the epithelium. When a specific odor is dissolved in mucus, chemical compounds will 
TABLE 1: Some prominent compounds present in tea aroma $[37,38,40]$.

\begin{tabular}{|c|c|c|c|}
\hline & Compound & Aroma type & Biosynthetic pathway \\
\hline (1) & (Z)-3-Hexenol & Green & Lipids \\
\hline (2) & Hexanal & Grassy, green & Lipid \\
\hline (3) & (Z)-4-Heptanal & Hay-like & Lipid \\
\hline (4) & (E)-2-Hexenal & Green & Lipid \\
\hline (5) & Hexanoic acid & Sweaty, green & Lipid \\
\hline (6) & $\beta$-Ionone & Woody, violet & Carotenoids \\
\hline (7) & $\alpha$-Ionone & Woody hay-like & Carotenoids \\
\hline (8) & Geranylacetone & Floral, hay-like & Carotenoids \\
\hline (9) & cis-Jasmone & Floral, jasmin-like & Lipids \\
\hline (10) & Methyljasmonate & Floral & Lipids \\
\hline (11) & (E)-Geraniol & Rose-like & Glycosides \\
\hline (12) & Linalool & Floral & Carotenoids, glycosides \\
\hline (13) & Linalool oxide I II III IV & Earthy, floral & Glycosides \\
\hline (14) & Methyl salicylate & Mint & Glycosides \\
\hline (15) & $\beta$-Damascenone & Fruity, apple-like & Carotenoids, glycosides \\
\hline (16) & Benzyl alcohol & Burning taste, faint aromatic & Glycosides \\
\hline (17) & 2-Phenyl ethanol & Honey-like & Glycosides \\
\hline (18) & 4-Hydroxy-2,5-dimethyl-3(2H)-furanone & Caramel-like & Glycosides \\
\hline (19) & 2-Ethyl-3,5-dimethylpyrazine & Nutty & Maillard reaction \\
\hline (20) & 5-Ethyl-2,3-dimethylpyrazine & Nutty & Maillard reaction \\
\hline$(21)$ & 2-Acetyl-2-thiazoline & Popcorn-like & Maillard reaction \\
\hline$(22)$ & Phenyl-acetaldehyde & Honey-like & Maillard reaction \\
\hline
\end{tabular}

bind with specific proteins in the receptor cells. The strength of binding differs from chemical to chemical thus creating a unique pattern of a signal which is sent to the brain as an electrical response. The brain identifies the particular order recalling memory of a similar smell [57].

Inspired by nature, electronic nose devices are fabricated to identify a particular aroma profile. Such a system consists of an array of gas sensors, where a combined response is evaluated as the aroma profile. There is a significant level of aroma produced during tea manufacturing which can be smelled even along the roadside of the factory. Therefore, as a quality controlling device, "e-nose" systems were developed by many research groups. The most comprehensive contribution for the study of the fermentation stage in black tea manufacturing is given by Ghosh et al. where they have developed e-nose, e-vision, and electronic tongue techniques to monitor the tea quality $[19,25,58]$. Then, research by Tozlu and coworkers has presented adapting e-nose technology to the factory level, real-time monitoring [26, 27]. Furthermore, use of e-nose systems to monitor the fermentation stage has also been conducted using commercially available e-nose systems recently [59].

A typical "e-nose" system contains an array of different gas sensors and vacuum pumps to collect aroma with programmable sequence control [27, 31]. The sensor array is placed in an airtight chamber which has inlets to introduce either the gas to be analyzed or the reference gas and an outlet. Most of the studies used metal oxide semiconductor (MOS) sensors for the multiple sensor arrays to classify the tea aroma. MOS are characterized by high sensitivity and mechanical robustness and can be continuously operated over a long time period [60]. The sensing element is based on tin dioxide $\left(\mathrm{SnO}_{2}\right)$. It has low conductivity in clean air. In the presence of detectable vapor, the sensor conductivity increases, depending on the concentration of odor molecules in the vapor. The sensor output is processed by a signal conditioning circuit for signal amplification, buffering, and signal conversion [36]. Sensor signals are converted into electrical signals, and data are interpreted using a developed algorithm. Table 2 indicates the summary of different gas sensors used in "e-nose" systems which are used in monitoring black tea fermentation.

Most "e-nose" systems contained several gas sensors which detect alcohols, Volatile Organic Compounds (VOC), liquefied petroleum (LP) gas, carbon monoxide (CO), and ammonia. In these systems, the highest response towards tea aroma was indicated by TGS 832, 823, and 2620 sensors which are commonly used to detect organic solvent gases [19, 22, 25, 29, 31]. This result agrees well with the chemical composition of tea aroma as listed in Table 1. But other types of sensors are also responding to the tea aroma as well. Therefore, the responses from multiple sensors are analyzed using an appropriate statistical method for further evaluation. Apart from this, custom-made devices [19, 27, 61] are also used to monitor the fermentation process [59]. Furthermore, e-nose systems have been used for classification of tea varieties as well [62].

2.4. Sniffing Process. The e-nose systems operate similar to breathing where an automated set of internal operations are 
TABLE 2: Summary of different gas sensors used in the e-nose system.

\begin{tabular}{|c|c|c|}
\hline Sensor Id & Main compound & References \\
\hline MQ 7 & $\mathrm{CO}, \mathrm{H}_{2}, \mathrm{LPG}, \mathrm{CH}_{4}$, alcohol & \multirow{6}{*}[18]{} \\
\hline MQ 3 & Alcohol, benzene, hexane, LPG, $\mathrm{CO}, \mathrm{CH}_{4}$ & \\
\hline MQ 4 & LPG, $\mathrm{CH}_{4}, \mathrm{H}_{2}$, CO, alcohol, smoke & \\
\hline MQ 6 & LPG, $\mathrm{H}_{2}, \mathrm{CH}_{4}, \mathrm{CO}$, alcohol & \\
\hline MQ 9 & LPG, $\mathrm{CO}, \mathrm{CH}_{4}$ & \\
\hline MQ 8 & $\mathrm{H}_{2}$, alcohol, $\mathrm{CO}, \mathrm{CH}_{4}$ & \\
\hline TGS 832 & $\mathrm{R}-12, \mathrm{R}-22, \mathrm{R}-134 \mathrm{a}$ & {$[22,25,29,31,63]$} \\
\hline TGS 823 & Methane, CO, isobutane, n-hexane, benzene, ethanol, acetone & {$[22,25,29,63,64]$} \\
\hline TGS 831 & $\mathrm{R}-21, \mathrm{R}-22$ & {$[25,31,63]$} \\
\hline TGS 816 & $\mathrm{CO}, \mathrm{CH}_{4}$, ethanol, propane, iso-butane, $\mathrm{H}_{2}$ & {$[25,63,64]$} \\
\hline TGS 2600 & Methane, $\mathrm{CO}$, isobutane, ethanol, $\mathrm{H}_{2}$ & {$[22,25,29,31,63]$} \\
\hline TGS 2610 & Ethanol, $\mathrm{H}_{2}$, isobutane, methane, propane & {$[22,25,29,31,63]$} \\
\hline TGS 2611 & Ethanol, $\mathrm{H}_{2}$, isobutane, methane & {$[22,25,29,31,63]$} \\
\hline TGS 2620 & $\mathrm{CH}_{4}, \mathrm{CO}$, isobutane, $\mathrm{H}_{2}$, ethanol & {$[25,28]$} \\
\hline
\end{tabular}

conducted during a test cycle, namely, (1) sensor warming up, (2) sampling, (3) purging, and (4) cleaning the sensor chamber before starting the next test cycle. At the first stage, sensors are warming up for some time. It optimizes the saturation of the sensor head space. Therefore, adequate sensor response can be obtained for the sampling process. The sampling process is conducted by exposing sensor array to a constant flow of volatile compounds through the pipe. During the cleaning operation, the sensor chamber is cleared with a blow of fresh air. Therefore, sensor heads go back to the baseline values. Typical sniffing cycle duration is 3-5 minutes in most of the e-nose systems developed so far. Figure 1 indicates the response of a typical sniffing cycle.

2.5. Validating E-Nose Response for Tea Fermentation. Quality checking using an e-nose system was done in using tea infusion preparation [30], sniffing aroma of fresh tea leaf particles placed in a container [64], or directly sniffing aroma from the production line as fermentation progresses [26, 27]. In these studies, e-nose response is validated or compared with the response obtained from human, or measuring TF/TR ratio, colorimetric methods, and good correlation to sensor response to pure aroma compounds were obtained in most studies $[27,36]$. A recent study has introduced a new fermentation band system to function with the e-nose system [27]. A simple colorimetric method is frequently used in tea factories to observe the progress of fermentation. There two distinct bands are observed which later correspond to the "first" and "second nose" in aroma. The studies conducted by Bhattacharyya and coworkers have clearly recorded a similar trend with the e-nose systems they developed [31].

2.6. Sensitivity Evaluation of E-Nose Sensors. Metal oxide sensors are subjected to the different chemicals that are present in black tea to evaluate the sensitivity of the sensors. A particular amount of each chemical is taken during the sensor evaluation. Then, $\Delta \mathrm{Rs} / \mathrm{Rs}$ values of the sensors, where $\Delta \mathrm{Rs}$ is the change in resistance of the sensor and Rs is the base resistance value of the respective sensor, are calculated
[36]. The base resistance value may interfere if aroma is not removed properly from the sensor chamber. Therefore, reference gas is introduced to the chamber before the next sniffing cycle. Common reference gases used are oxygen [27], nitrogen [65], and fresh air [31].

\section{E-Nose Data Analysis}

PC-based data acquisition and automated operation were controlled by specially designed software using LabVIEW $[27,30,64]$. In this case, minimum human intervention is needed to use a graphical user interface, yet basic computer knowledge is needed to handle the instrument.

3.1. Data Processing. In most of the e-nose devices, sensor raw response is recorded with time. There are many factors such as efficiency of the pumping system, noisy gas sensor recordings, and retention of sample aroma after the cleaning stage that influence the sensor response. Therefore, raw data are pretreated using single value decomposition (SVD) or using a difference model where change in sensor resistance with respect to a reference air is calculated and then entire $\mathrm{dR}$ data set is normalized by maximum value to set range to (0-1) [30]. In another study, standard normal variate (SNV) is used for data preprocessing with commercial devices [66]. Furthermore, feature extraction methods are also used in e-nose signal preprocessing [65].

3.2. Methodologies Used in Finding the Optimum Fermentation Time. Attempts to find the optimum fermentation time for black tea processing were conducted using unsupervised and supervised pattern recognition methods. Tea classification from e-nose data is complex as the emission of volatile compounds from tea leaves varies with time. Singular Value Decomposition (SVD) technique was used to analyze the peaks of sensor outputs [67]. In some studies, data were processed using Principal Component Analysis (PCA) and Fuzzy C Means (FCM) algorithm [61], and then, 2-Norm Method (2-NM) and Mahalanobis distance method 


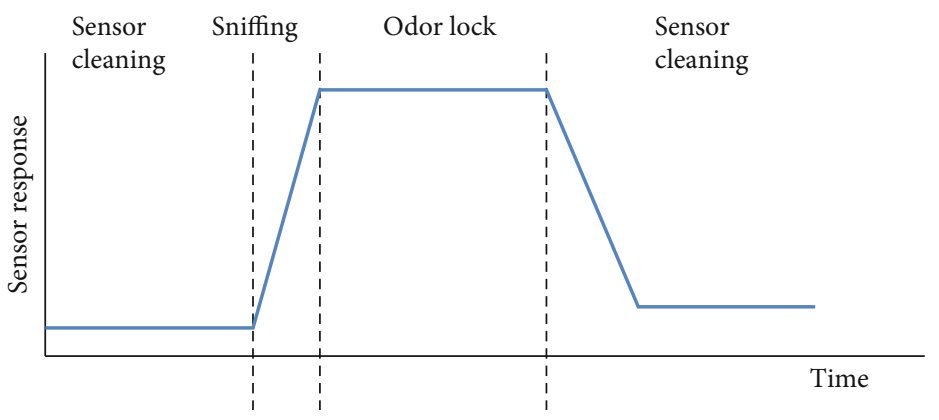

Figure 1: A sample sniffing cycle.

(MDM) have been used to analyze data [67]. Linear Discriminant Analysis (LDA), Back Propagation Multilayer Perceptron (BP-MLP), Bayes, and KNN-3 methods were used for the odor classification; the self-organizing map (SOM) method along with Radial Basis Function (RBF) network and Probabilistic Neural Network (PNN) classifier $[27,30,67]$ were also used for the odor classification where PNN produced best results [67]. MATLAB software was used to perform these techniques. In [27], the KNN-3 method has given the best accuracy with $74.19 \%$ classification performance achieved in [28]. Using FCM and SOM feature extraction techniques along with the RBF neural network, it has achieved $100 \%$ correct classification for five different tea samples, each of which has different qualities [30].

When analyzing e-nose data, a scoring method based on 2-NM and MDM has shown similar behavior to the colorimetric variation of tea during fermentation [31]. However, when considering updating the tea fermentation data, the Radial Basis Function (RBF) neural network method was used to incorporate new data acquired while keeping the previous data [29]. The data analysis component was further developed by Battacharya and coworkers so that the optimum fermentation level can be predicted with the initial set of data collected while fermentation is ongoing. This prediction of optimum fermentation time is useful to maintain constant quality of tea during production.

However, there are many challenges when adopting an e-nose system to monitor the fermentation process in real time. The work of Tozlu and Okumus has developed an enose system so that fermentation can be monitored at the production line [27]. A different approach was introduced by Sharma and coworkers where eight quartz crystal microbalance (QCM) sensors were used in construction of the e-nose system. The sensors are coated with different glucose derivatives with polyethylene glycol (PEG) with varying molecular weights. A comparison of QCM results with traditional colorimetric methods indicated good agreement suggesting it as a promising method to detect the optimum fermentation level [68].

\section{Conclusions}

Aroma, color, and taste are the most common testing quality parameters of tea. These quality parameters are highly dependent on the fermentation stage in the black tea manufacturing process. Therefore, finding the optimum fermentation time is crucial to maintain consistent highquality tea. In this review, use of e-nose systems to monitor the fermentation stage in black tea manufacturing is discussed. The e-nose systems consist of an array of gas sensors which are commercially used to monitor alcohol, methane, hydrogen, LPG, VOC, ammonia, carbon monoxide, and smoke. The combined response of these sensors is used in classification, quantification, and prediction of the level of aroma during the fermentation stage. Here, both unsupervised and supervised classification techniques are used to distinguish aroma profile generated during different time intervals. The results obtained in e-nose systems developed so far are capable of identifying the "first" and "second" smell peaks with the aid of a statistical method. However, the current systems developed require insight into the following perspectives for further development.

(i) In most systems, the sample is isolated from the production environment when aroma levels are detected. However, this is not practical when adapting to the real production line. Care should be taken to minimize the influence from the adjacent fermentation beds when adapting to factory conditions

(ii) Most studies have not addressed which level of dhool is used. Depending on the dhool number, fermentation duration is set in such that dhool 1 has the longest fermentation period and big bulk or dhool 4 will have the shortest fermentation time. The future system should be able to distinguish the aroma level based on the dhool number as well

(iii) All studies have been conducted under controlled weather conditions. However, in real factory conditions, weather and temperatures are dynamic and even show 10-15 degrees centigrade difference between morning batches to noon batches. Thus, statistical analysis should incorporate correlations with humidity and temperature data

\section{Conflicts of Interest}

The authors declare that there is no conflict of interest regarding the publication of this paper. 


\section{Acknowledgments}

The authors would like to thank the National Research Council of Sri Lanka (NRC 17-038) and University of Sri Jayewardenepura, Sri Lanka (ASP/01/RE/SCI/2019/28), for their funding support.

\section{References}

[1] K. Chang, World tea economy: trends and opportunities, pp. 1$14,2016$.

[2] P. O. Owuor and M. Obanda, "Clonal variations in the response of hard physically withered leaf to rehydration following long chemical wither durations," International Journal of Tea Science, vol. 13, no. 1-2, 2016.

[3] S. Saranka, T. Kartheeswaran, D. D. C. Wanniarachchi, and W. Wanniarachchi, "Monitoring fermentation of black tea with image processing techniques," Proc. Tech. Sess., vol. 32, pp. 31-37, 2016.

[4] L. P. Bhuyan, S. Sabhapondit, B. D. Baruah, C. Bordoloi, R. Gogoi, and P. Bhattacharyya, "Polyphenolic compounds and antioxidant activity of CTC black tea of North-East India," Food Chemistry, vol. 141, no. 4, pp. 3744-3751, 2013.

[5] M. Obanda, P. Okinda Owuor, and R. Mang'oka, "Changes in the chemical and sensory quality parameters of black tea due to variations of fermentation time and temperature," Food Chemistry, vol. 75, no. 4, pp. 395-404, 2001.

[6] W. A. J. M. De Costa, A. J. Mohotti, and M. A. Wijeratne, "Ecophysiology of tea," Brazilian Journal of Plant Physiology, vol. 19, no. 4, pp. 299-332, 2007.

[7] L. Emdadi, B. Nasernajad, and S. T. Shokrgozar, "Optimization of withering time and fermentation conditions during the manufacture of black tea using a response surface methodology," Scientia Iranica, vol. 16, no. 1, 2009.

[8] P. Tang, D.-Y. Shen, Y.-Q. Xu, X.-C. Zhang, J. Shi, and J.F. Yin, "Effect of fermentation conditions and plucking standards of tea leaves on the chemical components and sensory quality of fermented juice," Journal of Chemistry, vol. 2018, Article ID 4312875, 7 pages, 2018.

[9] T. Muthumani and R. S. S. Kumar, "Influence of fermentation time on the development of compounds responsible for quality in black tea," Food Chemistry, vol. 101, no. 1, pp. 98-102, 2007.

[10] K. R. Jolvis Pou, "Fermentation: the key step in the processing of black tea," Journal of Biosystems Engineering, vol. 41, no. 2, pp. 85-92, 2016.

[11] E. A. H. Roberts and R. F. Smith, "Spectrophotometric measurements of theaflavins and thearubigins in black tea liquors in assessments of quality in teas," Analyst, vol. 86, no. 1019, p. 94, 1961.

[12] S. Borah and M. Bhuyan, "Quality indexing by machine vision during fermentation in black tea manufacturing," in Proceedings Volume 5132, Sixth International Conference on Quality Control by Artificial Vision, Gatlinburg, TE, United States, 2003.

[13] S. Borah, Machine vision for tea quality monitoring with special emphasis on fermentation and grading emphasis on fermentation and grading, p. 178, 2005.

[14] G. Singh and N. Kamal, "Machine vision system for tea quality determination -tea quality index (TQI), "IOSR Journal of Engineering, vol. 3, no. 7, pp. 46-50, 2013.
[15] Y.-Q. Yang, H.-X. Yin, H.-B. Yuan, Y.-W. Jiang, C.-W. Dong, and Y.-L. Deng, "Characterization of the volatile components in green tea by IRAE-HS-SPME/GC-MS combined with multivariate analysis," PLoS One, vol. 13, no. 3, article e0193393, pp. 1-19, 2018.

[16] G. S. Gill, A. Kumar, and R. Agarwal, "Monitoring and grading of tea by computer vision - a review," Journal of Food Engineering, vol. 106, no. 1, pp. 13-19, 2011.

[17] S. Borah, M. Bhuyan, and H. Saikia, ANN based colour detection in tea fermentation, 2002.

[18] J. B. Lazaro, A. Ballado, F. P. F. Bautista, J. K. B. So, and J. M. J. Villegas, "Chemometric data analysis for black tea fermentation using principal component analysis," in AIP Conference Proceedings, pp. 1-6, Location- Bandung, Indonesia, December 2018.

[19] S. Ghosh, N. Bhattacharyya, B. Tudu, and R. Bandyopadhyay, "Electronic nose for on-line quality evaluation of black tea using incremental SOM techniques," in 2015 2nd International Symposium on Physics and Technology of Sensors (ISPTS), Pune, India, March 2015.

[20] Z. Qin, X. Pang, D. Chen, H. Cheng, X. Hu, and J. Wu, "Evaluation of Chinese tea by the electronic nose and gas chromatography-mass spectrometry: correlation with sensory properties and classification according to grade level," Food Research International, vol. 53, no. 2, pp. 864-874, 2013.

[21] H. Jiang and Q. Chen, "Development of electronic nose and near infrared spectroscopy analysis techniques to monitor the critical time in SSF process of feed protein," Sensors, vol. 14, no. 10, pp. 19441-19456, 2014.

[22] B. T. Ghosh, N. Bhattacharyya, and R. Bandyopadhyay, "A recurrent Elman network in conjunction with an electronic nose for fast prediction of optimum fermentation time of black tea," Neural Computing and Applications, vol. 31, Supplement 2, pp. 1165-1171, 2019.

[23] H. Li, B. Zhang, W. Hu, Y. Liu, C. Dong, and Q. Chen, "Monitoring black tea fermentation using a colorimetric sensor array-based artificial olfaction system," Journal of Food Processing and Preservation, vol. 42, no. 1, article e13348, 2018.

[24] H. Yu, Y. Wang, and J. Wang, "Identification of tea storage times by linear discrimination analysis and back-propagation neural network techniques based on the eigenvalues of principal components analysis of E-nose sensor signals," Sensors, vol. 9, no. 10, pp. 8073-8082, 2009.

[25] M. Sharma, D. Ghosh, and N. Bhattacharya, "Electronic nose a new way for predicting the optimum point of fermentation of black tea," International Journal of Engineering and Science Invention, vol. 2, no. 3, pp. 56-60, 2013.

[26] B. Tozlu, H. İ. Okumuş, C. Şimşek, and Ö. Aydemir, “On-line monitoring of theaflavins and thearubigins ratio in Turkish black tea using electronic nose," International Journal of Scientific Engineering and Applied Science, vol. 7, no. 5, pp. 23058269, 2015.

[27] B. H. Tozlu and H. İ. Okumuş, "A new approach to automation of black tea fermentation process with electronic nose," Automatika, vol. 59, no. 3-4, pp. 373-381, 2018.

[28] B. Tozlu, H. I. Okumus, and C. Simsek, "Online quality classifying with electronic nose for black tea production," International Journal of Academic Research, vol. 6, no. 4, 2014.

[29] B. Tudu, A. Jana, A. Metla, D. Ghosh, N. Bhattacharyya, and R. Bandyopadhyay, "Electronic nose for black tea quality 
evaluation by an incremental RBF network," Sensors and Actuators B: Chemical, vol. 138, no. 1, pp. 90-95, 2009.

[30] R. Dutta, K. R. Kashwan, M. Bhuyan, E. L. Hines, and J. W. Gardner, "Electronic nose based tea quality standardization," Neural Networks, vol. 16, no. 5-6, pp. 847-853, 2003.

[31] N. Bhattacharyya, S. Seth, B. Tudu et al., "Monitoring of black tea fermentation process using electronic nose," Journal of Food Engineering, vol. 80, no. 4, pp. 1146-1156, 2007.

[32] S. Buratti, A. Casiraghi, P. Minghetti, and G. Giovanelli, "The joint use of electronic nose and electronic tongue for the evaluation of the sensorial properties of green and black tea infusions as related to their chemical composition," Food and Nutrition Sciences, vol. 4, no. 6, pp. 605-615, 2013.

[33] M. Xu, J. Wang, and S. Gu, "Rapid identification of tea quality by E-nose and computer vision combining with a synergetic data fusion strategy," Journal of Food Engineering, vol. 241, pp. 10-17, 2019.

[34] R. Banerjee, P. Chattopadhyay, R. Rani, B. Tudu, R. Bandyopadhyay, and N. Bhattacharyya, "Discrimination of black tea using electronic nose and electronic tongue: a Bayesian classifier approach," in 2011 International Conference on Recent Trends in Information Systems, Kolkata, India, December 2011.

[35] R. Banerjee, B. Tudu, L. Shaw, A. Jana, N. Bhattacharyya, and R. Bandyopadhyay, "Instrumental testing of tea by combining the responses of electronic nose and tongue," Journal of Food Engineering, vol. 110, no. 3, pp. 356-363, 2012.

[36] N. Bhattacharyya, R. Bandyopadhyay, M. Bhuyan, B. Tudu, D. Ghosh, and A. Jana, "Electronic nose for black tea classification and correlation of measurements with "tea taster" marks," IEEE Transactions on Instrumentation and Measurement, vol. 57, no. 7, pp. 1313-1321, 2008.

[37] Z. Yang, S. Baldermann, and N. Watanabe, "Recent studies of the volatile compounds in tea," Food Research International, vol. 53, no. 2, pp. 585-599, 2013.

[38] X. Q. Zheng, Q. S. Li, L. P. Xiang, and Y. R. Liang, "Recent advances in volatiles of teas," Molecules, vol. 21, no. 3, p. 338, 2016.

[39] H. Co and G. W. Sanderson, "Biochemistry of tea fermentation: conversion of amino acids to black tea aroma constituents," Journal of Food Science, vol. 35, no. 2, pp. 160-164, 1970.

[40] C.-T. Ho, X. Zheng, and S. Li, "Tea aroma formation," Food Science and Human Wellness, vol. 4, no. 1, pp. 9-27, 2015.

[41] X. Chen, D. Chen, H. Jiang et al., "Aroma characterization of Hanzhong black tea (Camellia sinensis)_ using solid phase extraction coupled with gas chromatography -mass spectrometry and olfactometry and sensory analysis," Food Chemistry, vol. 274, pp. 130-136, 2019.

[42] V. Sai, P. Chaturvedula, and I. Prakash, "The aroma, taste, color and bioactive constituents of tea," Journal of Medicinal Plants Research, vol. 5, no. 11, pp. 2110-2124, 2011.

[43] P. V. S. Kumar, S. Basheer, R. Ravi, and M. S. Thakur, "Comparative assessment of tea quality by various analytical and sensory methods with emphasis on tea polyphenols," Journal of Food Science and Technology, vol. 48, no. 4, pp. 440-446, 2011.

[44] N. Togari, A. Kobayashi, and T. Aishima, "Pattern recognition applied to gas chromatographic profiles of volatile components in three tea categories," Food Research International, vol. 28 , no. 5, pp. 495-502, 1995.
[45] L. Cao, X. Guo, G. Liu et al., "A comparative analysis for the volatile compounds of various Chinese dark teas using combinatory metabolomics and fungal solid-state fermentation," Journal of Food and Drug Analysis, vol. 26, no. 1, pp. 112123, 2018

[46] S. Baldermann, Z. Yang, T. Katsuno et al., "Discrimination of green, oolong, and black teas by GC-MS analysis of characteristic volatile flavor compounds," American Journal of Analytical Chemistry, vol. 5, no. 9, pp. 620-632, 2014.

[47] P. Pripdeevech, S. Moonggoot, S. Popluechai, and E. Chukeatirote, "Analysis of volatile constituents of fermented tea with Bacillus subtilis by SPME-GC-MS," Chiang Mai Journal of Science, vol. 41, no. 2, pp. 395-402, 2014.

[48] E. Sheibani, S. E. Duncan, D. D. Kuhn, A. M. Dietrich, J. J. Newkirk, and S. . F. O'Keefe, "Changes in flavor volatile composition of oolong tea after panning during tea processing," Food Science \& Nutrition, vol. 4, no. 3, pp. 456-468, 2016.

[49] S. K. Chandini, L. J. Rao, M. K. Gowthaman, D. J. Haware, and R. Subramanian, "Enzymatic treatment to improve the quality of black tea extracts," Food Chemistry, vol. 127, no. 3, pp. 1039-1045, 2011.

[50] A. Laddi, S. Sharma, A. Kumar, and N. R. Prakash, "Influence on color attributes of freshly brewed tea with time due to variations in temperature conditions," International Journal of Computer Applications, vol. 34, no. 7, pp. 7-9, 2011.

[51] A. E. Thea, M. A. Lloret, L. A. Brumovsky, and M. E. Schmalko, "Differences in quality parameters between types of commercial tea from Argentina," International Journal of Food Studies, vol. 2, pp. 168-178, 2012.

[52] D. L. Whitehead and C. M. Temple, "Rapid method for measuring thearubigins and theaflavins in black tea using C18 sorbent cartridges," Journal of the Science of Food and Agriculture, vol. 58, no. 1, pp. 149-152, 1992.

[53] T. Samanta, V. Cheeni, S. Das, A. B. Roy, B. C. Ghosh, and A. Mitra, "Assessing biochemical changes during standardization of fermentation time and temperature for manufacturing quality black tea," Journal of Food Science and Technology, vol. 52, no. 4, pp. 2387-2393, 2015.

[54] S.-D. Lv, Y. S. Wu, Y. Z. Song et al., "Multivariate analysis based on GC-MS fingerprint and volatile composition for the quality evaluation of Pu-Erh green tea," Food Analytical Methods, vol. 8, no. 2, pp. 321-333, 2015.

[55] A. Y. Yashin, B. V. Nemzer, E. Combet, and Y. I. Yashin, "Determination of the chemical composition of tea by chromatographic methods: a review," Journal of Food Research, vol. 4, no. 3, p. 56, 2015.

[56] H. Q. Tuan, N. D. Thinh, and N. T. Minh Tu, "Composition analysis of OTD and CTC black teas (Camellia sinensis) for the volatile profile differentiation by HS-SPME/GC-MS," Vietnam Journal of Science and Technology, vol. 54, no. 4, p. 483, 2016.

[57] W. Hu, L. Wan, Y. Jian et al., "Electronic noses: from advanced materials to sensors aided with data processing," Advanced Materials Technologies, vol. 4, no. 2, article 1800488, 2018.

[58] A. Akuli, A. Pal, G. Bej et al., "A machine vision system for estimation of theaflavins and thearubigins in orthodox black tea," International Journal of Smart Sensing and Intelligent Systems, vol. 9, no. 2, pp. 709-731, 2016.

[59] N. Manigandan, V. A. S. And, and P. Surendar, "Handheld electronic nose (HEN) for detection of optimum fermentation time during tea manufacture and assessment of tea quality," 
International Journal of Advanced Research, vol. 7, no. 7, pp. 697-702, 2019.

[60] Z. Yunusa, Sensors \& transducers gas sensors: a review, 2014.

[61] R. Dutta, E. L. Hines, J. W. Gardner, K. R. Kashwan, and M. Bhuyan, "Tea quality prediction using a tin oxide-based electronic nose: an artificial intelligence approach," Sensors and Actuators B: Chemical, vol. 94, no. 2, pp. 228-237, 2003.

[62] S. N. Hidayat, K. Triyana, I. Fauzan et al., "The electronic nose coupled with chemometric tools for discriminating the quality of black tea samples in situ," Chemosensors, vol. 7, no. 3, p. 29, 2019.

[63] A. Ghosh, A. K. Bag, P. Sharma et al., "Monitoring the fermentation process and detection of optimum fermentation time of black tea using an electronic tongue," IEEE Sensors Journal, vol. 15, no. 11, pp. 6255-6262, 2015.

[64] N. Bhattacharyya, S. Seth, B. Tudu et al., "Detection of optimum fermentation time for black tea manufacturing using electronic nose," Sensors and Actuators B: Chemical, vol. 122, no. 2, pp. 627-634, 2007.

[65] R. Zhi, L. Zhao, and D. Zhang, "A framework for the multilevel fusion of electronic nose and electronic tongue for tea quality assessment," Sensors, vol. 17, no. 5, p. 1007, 2017.

[66] S. Kumar, P. C. Panchariya, P. B. Prasad, and A. L. Sharma, "Non-destructive classification of Assam black tea using ultra-fast gas chromatography (UFGC) coupled with soft independent modeling of class analogy (SIMCA)," Sensors and Transducers, vol. 186, no. 3, pp. 2014-2015, 2015.

[67] N. Bhattacharya, B. Tudu, A. Jana, D. Ghosh, R. Bandhopadhyaya, and M. Bhuyan, "Preemptive identification of optimum fermentation time for black tea using electronic nose," Sensors and Actuators B: Chemical, vol. 131, no. 1, pp. 110-116, 2008.

[68] P. Sharma, A. Ghosh, B. Tudu et al., "Monitoring the fermentation process of black tea using QCM sensor based electronic nose," Sensors and Actuators B: Chemical, vol. 219, pp. 146$157,2015$. 